\title{
Supplementary Material of
}

\section{Adaptive Baseline Finder, a statistical data selection strategy to identify atmospheric $\mathrm{CO}_{2}$ baseline levels and its application to European elevated mountain stations}

Ye Yuan ${ }^{1}$, Ludwig Ries ${ }^{2}$, Hannes Petermeier ${ }^{3}$, Martin Steinbacher ${ }^{4}$, Angel J. Gómez-Peláez ${ }^{5}$, Markus C. Leuenberger ${ }^{6}$, Marcus Schumacher ${ }^{7}$, Thomas Trickl ${ }^{8}$, Cedric Couret ${ }^{2}$, Frank Meinhardt $^{9}$, Annette Menzel ${ }^{1,10}$

${ }^{1}$ Department of Ecology and Ecosystem Management, Technische Universität München, Freising, Germany

${ }^{2}$ German Environment Agency (UBA), Zugspitze, Germany

${ }^{3}$ Department of Mathematics, Technische Universität München, Freising, Germany

${ }^{4}$ Empa, Laboratory for Air Pollution/Environmental Technology, Dübendorf, Switzerland

${ }^{5}$ Izaña Atmospheric Research Center, Meteorological State Agency of Spain (AEMET), Santa Cruz de Tenerife, Spain

${ }^{6}$ Climate and Environmental Physics Division, Physics Institute and Oeschger Centre for Climate Change Research, University of Bern, Bern, Switzerland

${ }^{7}$ Meteorological Observatory Hohenpeissenberg, Deutscher Wetterdienst (DWD), Hohenpeissenberg, Germany

${ }^{8}$ Institute of Meteorology and Climate Research, Atmospheric Environmental Research (IMK-IFU), Karlsruhe Institute of Technology (KIT), Garmisch-Partenkirchen, Germany

${ }^{9}$ German Environment Agency (UBA), Schauinsland, Germany

${ }^{10}$ Institute for Advanced Study, Technische Universität München, Garching, Germany

Correspondence to: Ye Yuan (yuan@wzw.tum.de) 


\section{S1 Applicability of ABF}

\section{S1.1 Running frequency of ABF}

We compared the $\mathrm{ABF}$ derived start time windows of overall running frequency with seasonal running frequency, shown in Table S1.1.

Table S1.1: Start time window by overall frequency and seasonal frequency (data of whole period), hours in advance and delayed are listed in brackets.

\begin{tabular}{lllll} 
Season & IZO & ZSF & SNB & JFJ \\
\hline Overall & 1 a.m. -6 a.m. & 10 p.m. -3 a.m. & 3 a.m. -8 a.m. & 11 p.m. -4 a.m. \\
\hline Winter & 2 a.m. -7 a.m. $(+1$ hr $)$ & 1 a.m. -6 a.m. $(+3$ hr $)$ & 6 p.m. -11 p.m. $(-9$ hr $)$ & 3 a.m. -8 a.m. $(+4$ hr $)$ \\
Spring & 11 p.m. -4 a.m. $(-2$ hr $)$ & 10 p.m. -3 a.m. $(0$ hr $)$ & 5 a.m. -10 a.m. $(+2$ hr $)$ & 6 p.m. -11 p.m. $(-5$ hr $)$ \\
Summer & 1 a.m. -6 a.m. $(0$ hr $)$ & 0 a.m. -5 a.m. $(+2$ hr $)$ & 3 a.m. -8 a.m. $(0$ hr $)$ & 1 a.m. -6 a.m. $(+2$ hr $)$ \\
Autumn & 1 a.m. -6 a.m. $(0$ hr $)$ & 9 p.m. -2 a.m. $(-1$ hr $)$ & 5 a.m. -10 a.m. $(+2$ hr $)$ & 0 a.m. -5 a.m. $(+1$ hr $)$ \\
\hline
\end{tabular}

As a result, most of the seasonal derived start time windows differ moderately from the overall derived start time windows. However, two exceptions have been observed, which are winter at SNB and spring at JFJ. Therefore, graphical examination was taken in Fig. S1.1 (a) and (b).

For winter time at SNB, the seasonal derived start time window is with the least standard deviation, but doesn't exhibit the minimal value from the diurnal cycle. For winter, we expect the most representative level of $\mathrm{CO}_{2}$ to be around the minimal values with relatively small standard deviations. Therefore, the overall derived start time window is more suitable to be the ideal start time window in this case.

Similar case happens to the spring time at JFJ. For spring, the vegetation activities have been already influenced the $\mathrm{CO}_{2}$ diurnal cycle. The start time window in this case is not suitable as the minimal values included. Again, the overall derived start time window is more practical. 


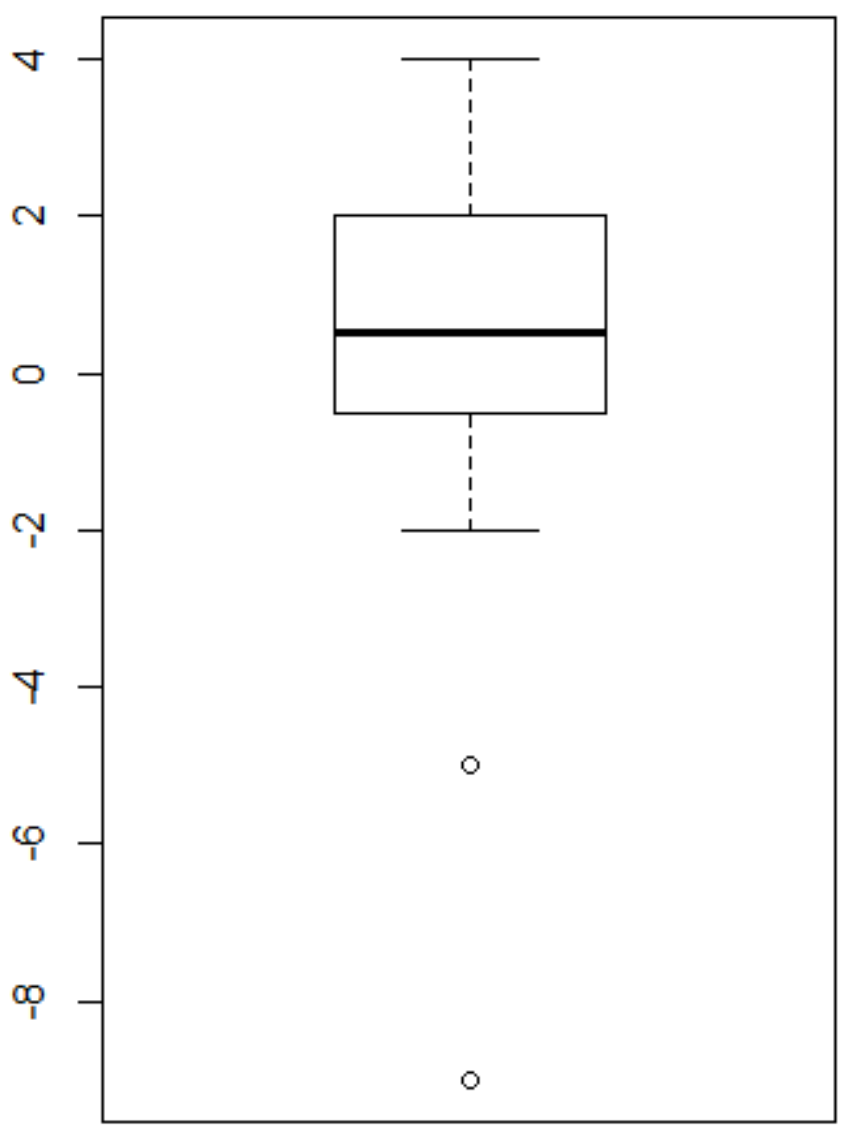

Figure S1.1 (a): Boxplot for hourly shifts of seasonal derived start time windows from overall derived start time windows, corresponding to Table S1.1. 

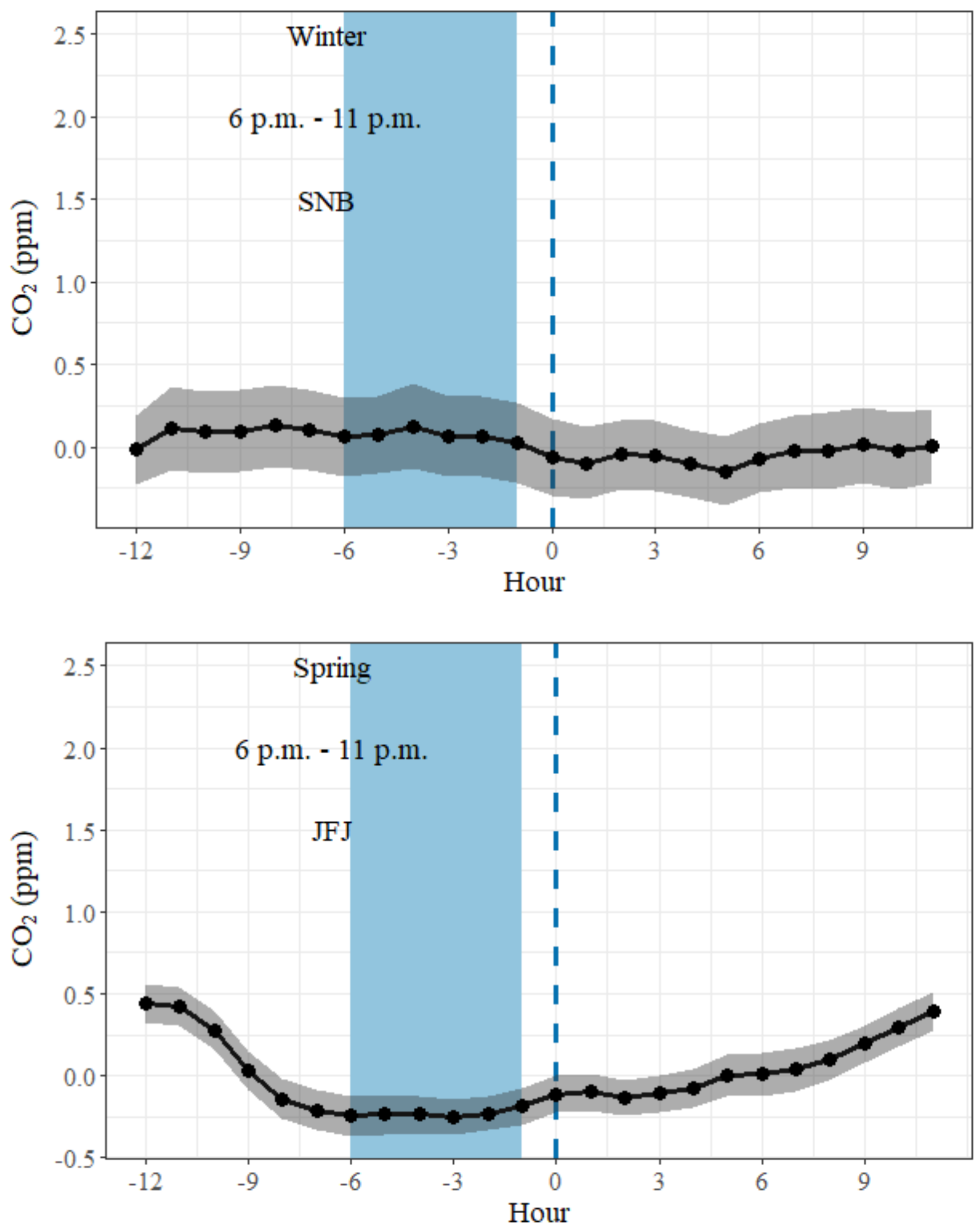

Figure S1.1 (b): Detrended mean diurnal variations of validated $\mathrm{CO}_{2}$ mole fractions (black) with $95 \%$ confidence intervals (grey) for winter time at SNB (upper) and for spring time at JFJ (below). The corresponding seasonal derived start time windows are shown both in blue shades and texts. 


\section{S1.2 Standard deviation threshold $s_{i, t h r e s h o l d}$}

The threshold of standard deviation determines the degree of variation for the selected time windows. In the study, we applied $0.3 \mathrm{ppm}$ to all the stations for inter-comparison. But it is clear that for low elevated stations like SSL, this value may be not appropriate. We tried $1.0 \mathrm{ppm}$ threshold for station SSL and resulted in the same start time window and a much higher selection percentage (26.14\% compared with $3.8 \%$ in the main text), however with a few irregular spikes observed below.

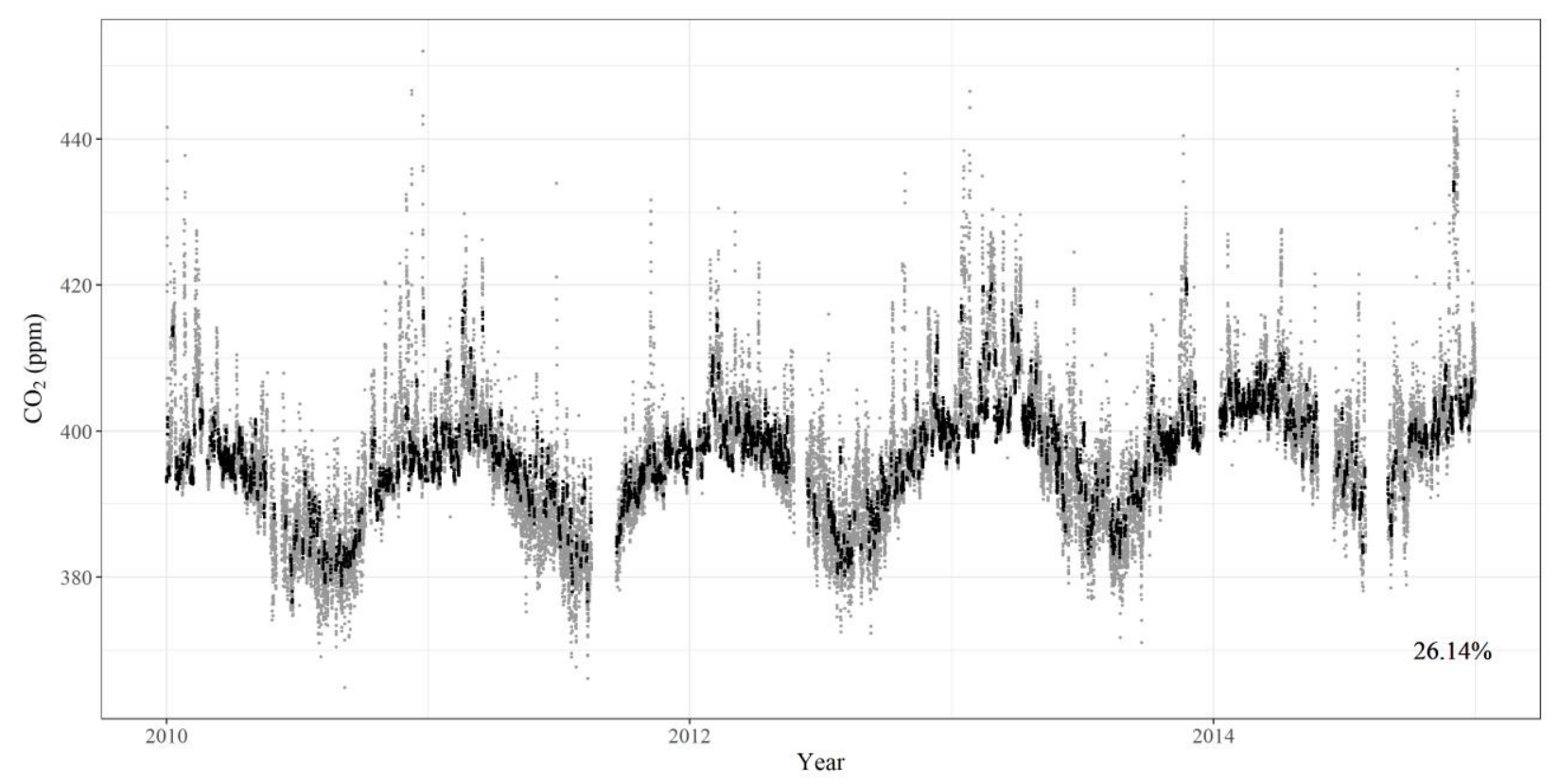

Figure S1.2: Time series plot of validated $\mathrm{CO}_{2}$ data set (grey) and ABF-selected data set (black) at SSL from 2010 to 2014. 


\section{S1.3 Time resolution $t r$}

Data at station ZSF were taken to evaluate the differences in data selection on different time resolution. To compare with the selected result based on hourly averages, we applied ABF directly to the 30-min validated data set. The resulting start time windows are the same, but the selection percentages are significantly different at $95 \%$ confidence interval, shown in table below.

Table S1.3 (a): Selection percentages by ABF applied to ZSF data sets.

\begin{tabular}{cc}
\hline Time resolution & ABF selection percentage $\pm \mathbf{9 5 \%}$ confidence interval \\
\hline 30-min & $13.46 \pm 0.22$ \\
1-hour & $14.76 \pm 0.33$ \\
\hline
\end{tabular}

For station JFJ, the 10-min validated data set was available for more detailed comparison. We prepared the ABFselected data sets on 10-min, 20-min and 30-min time scale to compare with the hourly selected results. The resulting start time windows are again the same. The results of selection percentages show again significant differences (at 95\% confidence interval) between each two data sets, shown in table below.

Table S1.3 (b): Selection percentages by ABF applied to JFJ data sets.

\begin{tabular}{cc}
\hline Time resolution & ABF selection percentage $\mathbf{\pm 9 5 \%}$ confidence interval \\
\hline 10-min & $18.73 \pm 0.14$ \\
20-min & $20.02 \pm 0.21$ \\
30-min & $20.75 \pm 0.26$ \\
1-hour & $22.14 \pm 0.37$ \\
\hline
\end{tabular}

As a result, these selection percentages clearly indicate a significantly smaller selection percentage with finer time resolution. This can be possibly explained based on the statistical property of ABF. While ABF evaluates the standard deviation within a time window, averaged data with larger time intervals show more statistical robustness. For $\mathrm{ABF}$, it is applicable for data sets with time resolution equal to or finer than one hour. 


\section{S2 Detrended diurnal cycles}
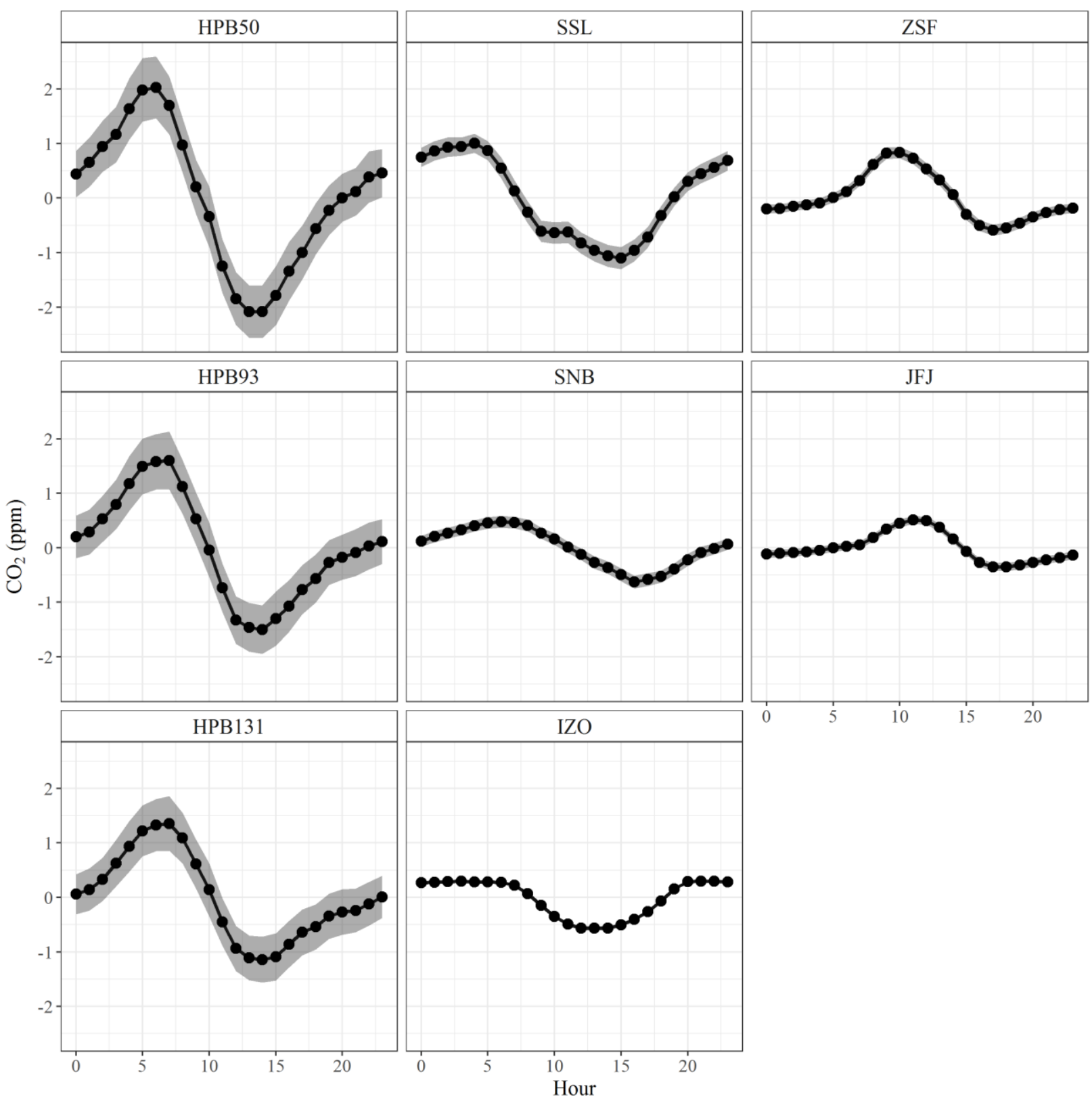

Figure S2: Detrended mean diurnal variation of validated $\mathrm{CO}_{2}$ mole fractions (black) with $95 \%$ confidence intervals (grey) at six European GAW stations. 


\section{S3 Selection percentages}

\section{S3.1 Table of selection percentages}

Table S3.1: List of selection percentages (\%) during ABF data selection process ( $\pi_{1}-$ selected days with valid start time window in all days; $\pi_{2}$ - selected hours with valid start time window in all hourly data; $\pi_{3}-$ selected hours after forward adaptive selection in all hourly data; $A B F$ - final selection percentages). The bottom shows the linear regression coefficients of stations (HPB is represented by HPB50; IZO is excluded) altitudes and the selection percentages at significance level of $0.05(* * *)$.

\begin{tabular}{lrrrr}
\hline Station ID & \multicolumn{1}{c}{$\boldsymbol{\pi}_{\mathbf{1}}$} & \multicolumn{1}{c}{$\boldsymbol{\pi}_{\mathbf{2}}$} & \multicolumn{1}{c}{$\boldsymbol{\pi}_{\mathbf{3}}$} & \multicolumn{1}{c}{$\mathbf{A B F}^{*}$} \\
\hline HPB50 & 15.2 & 2.1 & 2.6 & 3.2 \\
HPB93 & 22.7 & 3.2 & 3.9 & 4.8 \\
HPB131 & 29.6 & 4.3 & 5.2 & 6.2 \\
SSL & 14.0 & 2.6 & 3.1 & 3.8 \\
IZO & 85.2 & 20.0 & 26.1 & 36.2 \\
ZSF & 52.8 & 8.9 & 12.3 & 14.8 \\
SNB & 47.3 & 10.9 & 14.9 & 19.3 \\
JFJ & 69.3 & 12.1 & 17.4 & 22.1 \\
Linear regression coefficient $\left(\boldsymbol{\gamma}^{\mathbf{2}}\right)$ & $0.941^{* * *}$ & $0.996^{* * *}$ & $0.998^{* * * *}$ & $0.996^{* * * *}$ \\
\hline
\end{tabular}

*For $\mathrm{ABF}$, the final selection percentage is equivalent to the percentage of selected hours after backward adaptive selection in all hourly data.

S3.2 Linear regression of station altitudes and selection percentages

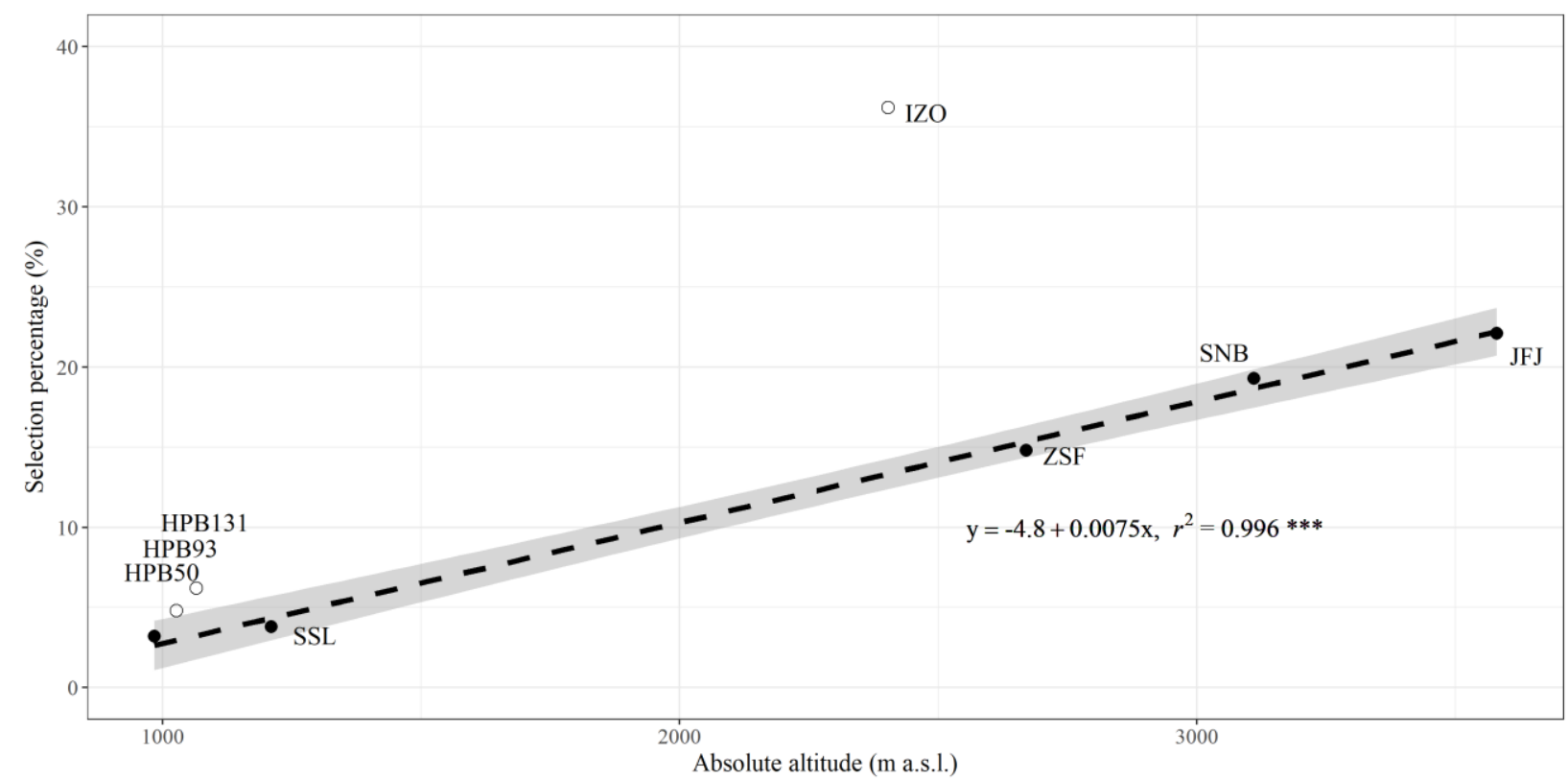

Figure S3.2: Linear regression between the absolute altitudes and the final selection percentages by ABF for all continental sites (excluding IZO). For HPB, only HPB50 is chosen as the demonstration sampling height. 
S3.3 Comparison of selection percentages among data selection methods

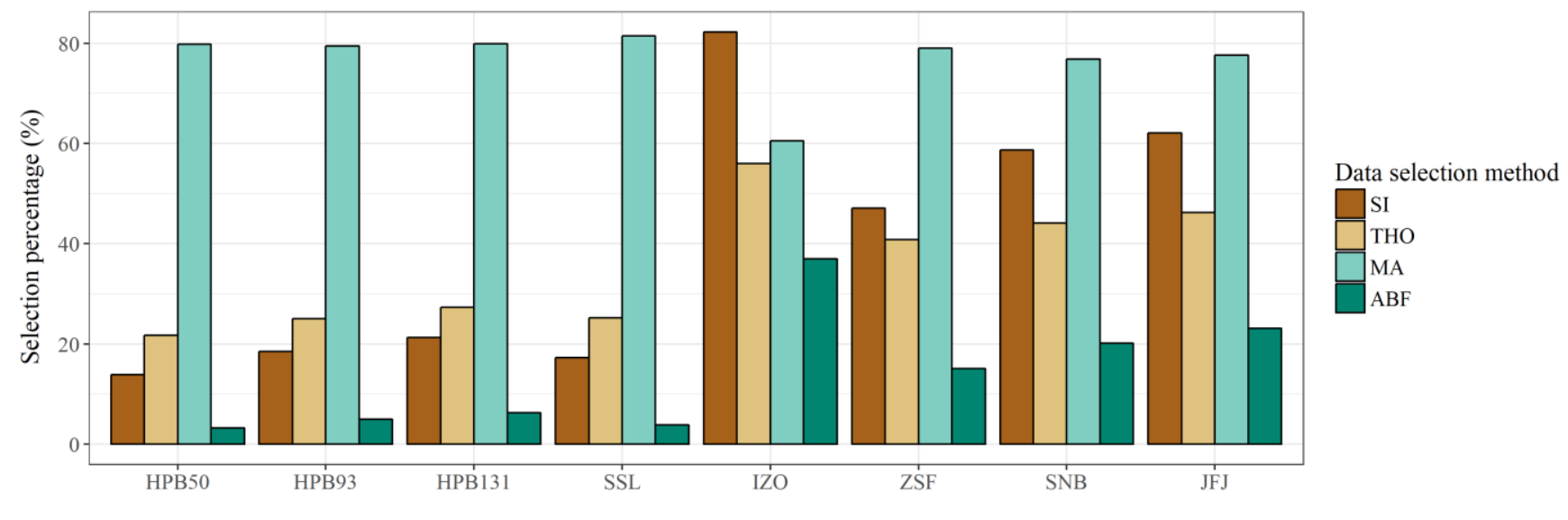

Figure S3.3: Comparison of the selection percentages by four statistical data selection methods (SI, THO, MA, ABF) applied to validated $\mathrm{CO}_{2}$ data sets at six GAW stations. 
S4 Mean monthly variations at SSL, SNB and JFJ

S4.1 SSL

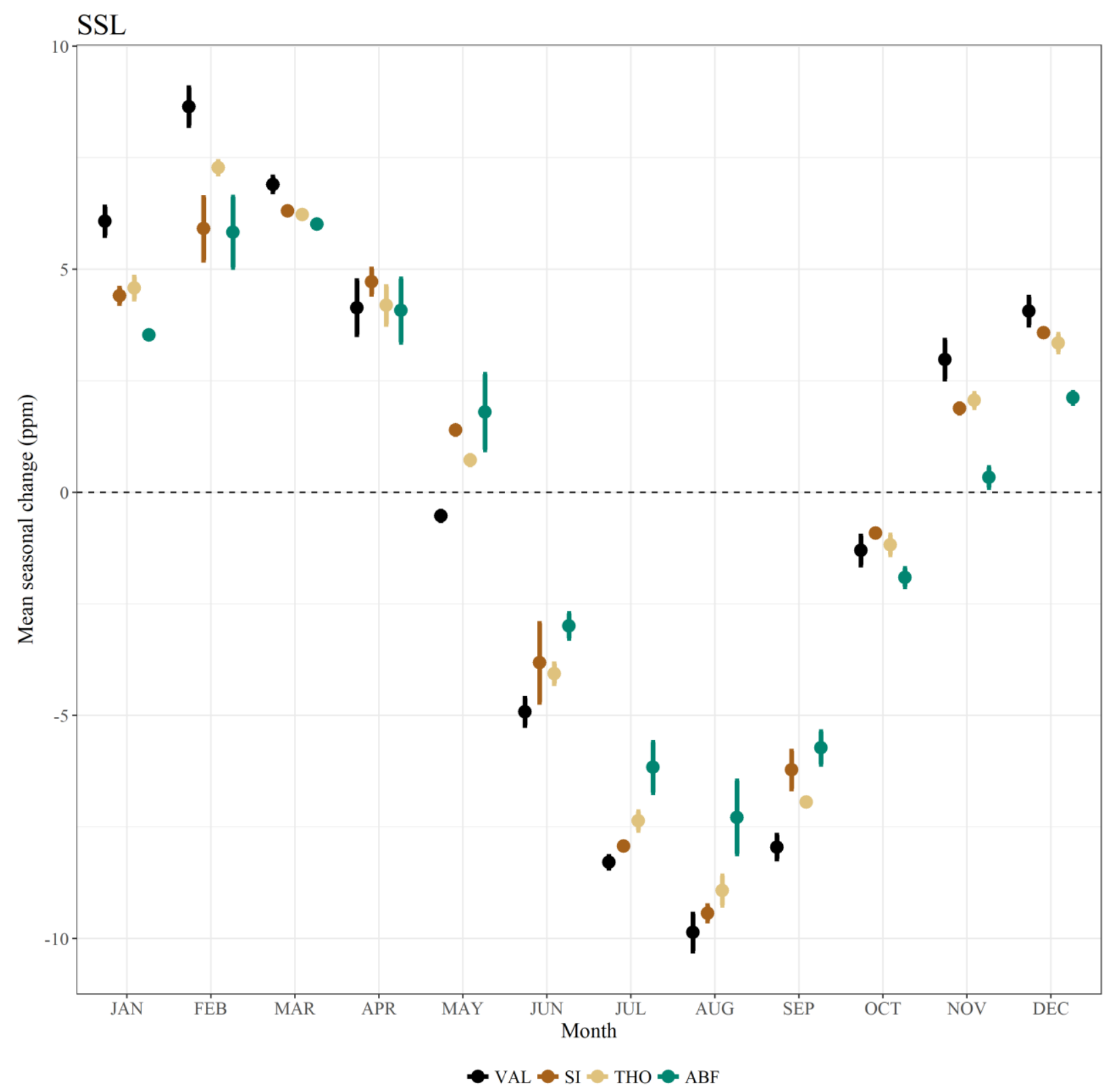

Figure S4.1: Mean monthly variation of the seasonal component decomposed by STL at SSL over the whole time period. 


\section{S4.2 SNB}

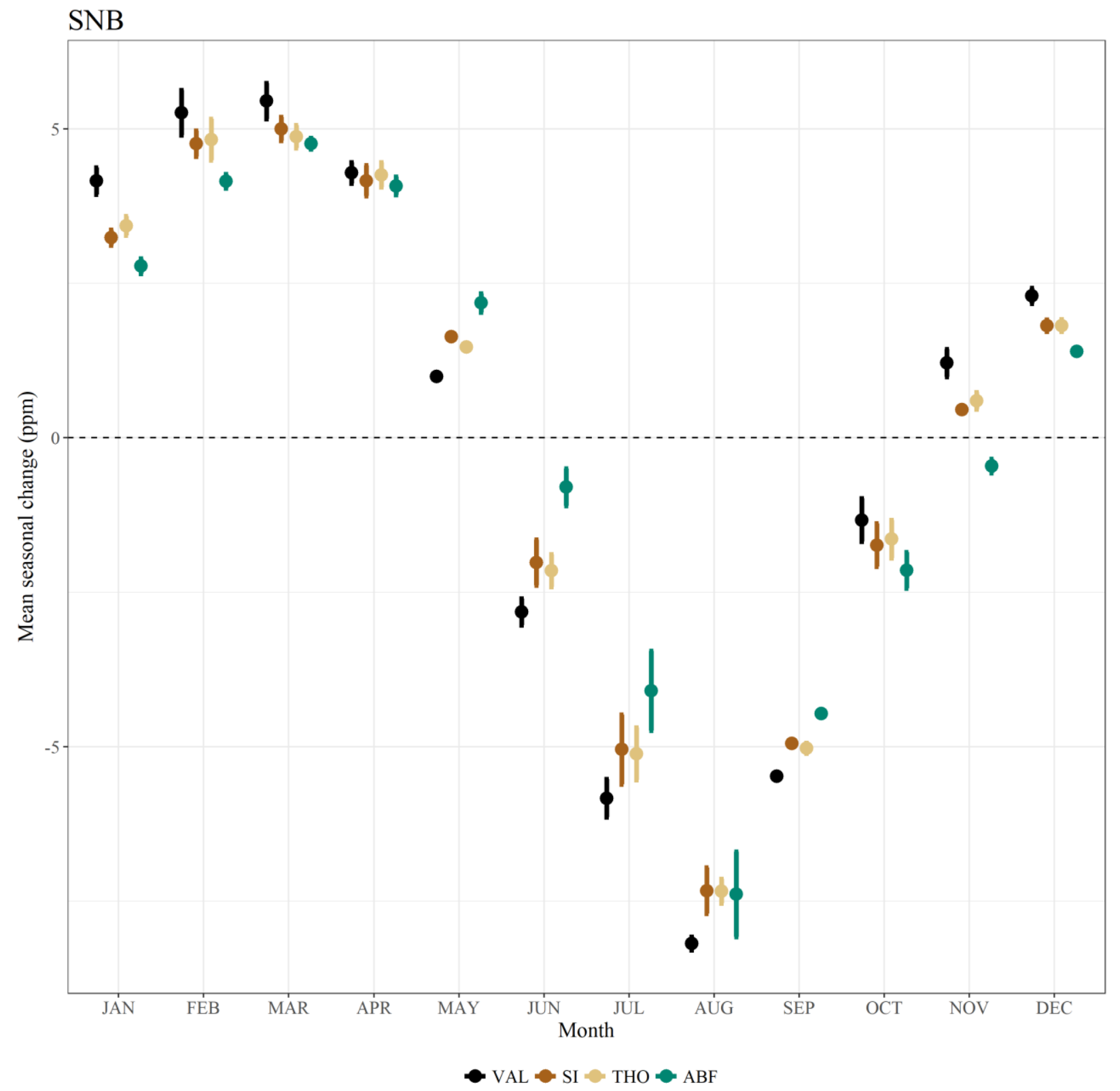

Figure S4.2: Mean monthly variation of the seasonal component decomposed by STL at SNB over the whole time period. 
S4.3 JFJ

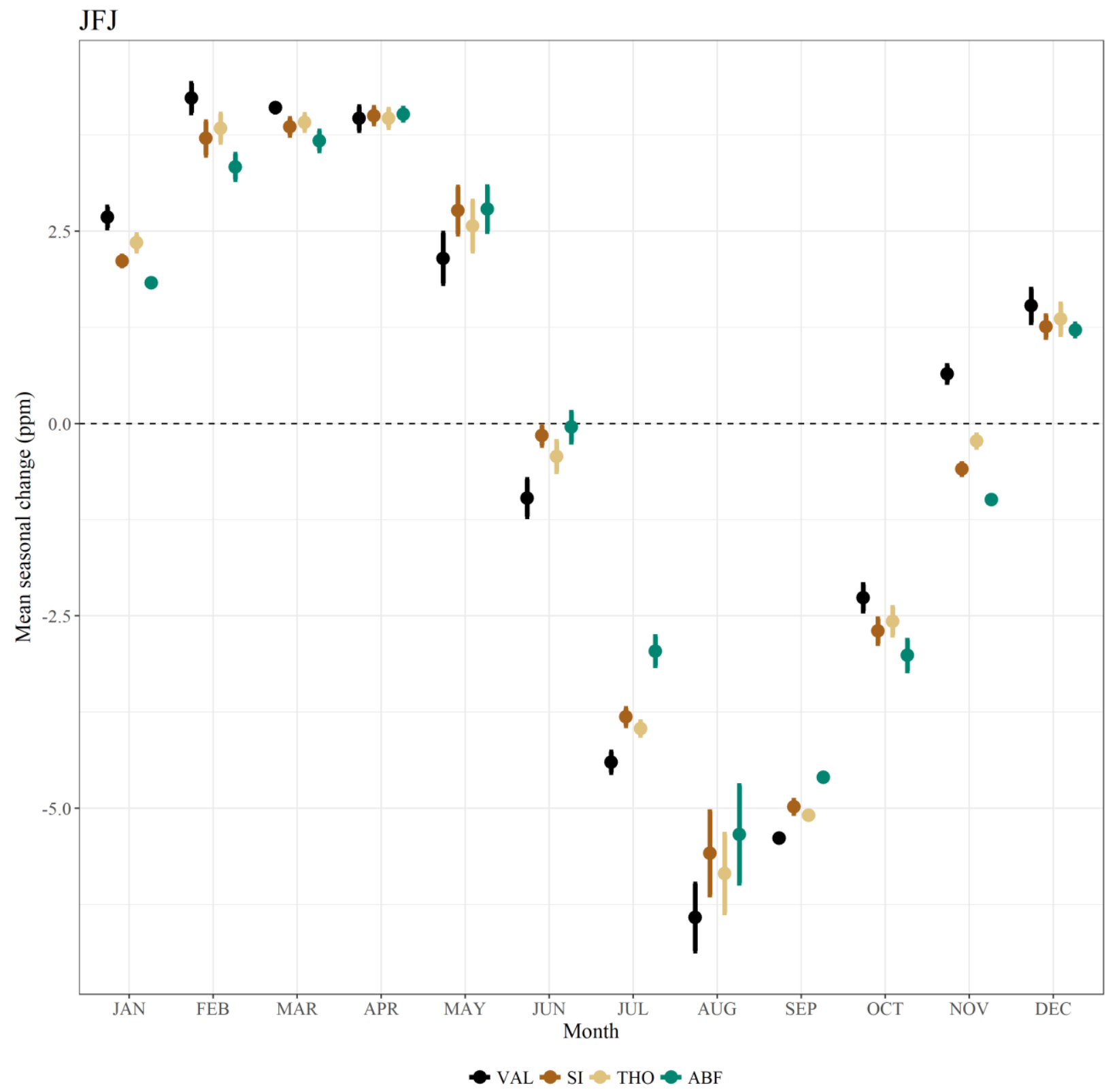

Figure S4.3: Mean monthly variation of the seasonal component decomposed by STL at JFJ over the whole time period. 